Le Débat, no 160, mai-août 2010, p. 151-160.

\title{
Bouleversements sur le front de la lecture
}

\author{
Christian Vandendorpe \\ Université d'Ottawa
}

Là je feuillette à cette heure un livre, à cette heure un aultre, sans ordre et sans desseing, à pièces décousues.

Montaigne, Essais, III, 3.

L'acte de lecture paraît si naturel qu'on a tendance à croire qu'il fait partie de l'organisation de base du cerveau humain, oubliant le temps qu'il a fallu pour maîtriser cette activité. Si l'histoire de la lecture ne s'était pas constituée comme discipline depuis moins d'un demi-siècle, nous pourrions encore penser que cette activité n'a guère varié au fil des âges. Or, on sait maintenant, entre autres choses, que les Romains lisaient à haute voix, que la lecture silencieuse ne s'est répandue en Europe que vers le XIIe siècle et que la passion pour la lecture de romans ne date que de quelques siècles.

La lecture est l'activité par laquelle le cerveau recueille des signes extérieurs et les interprète. Comme la perception des signes résulte ellemême d'un travail de construction, le cerveau s'adapte aux besoins de cet infatigable chasseur de signes qu'est l'homo sapiens et devient plus habile à repérer et comprendre les signes qui l'intéressent le plus souvent. Il y a donc réciprocité d'action entre le média et le contenu : loin d'être figées, nos connexions neuronales sont constamment réorganisées en fonction des activités cognitives que nous effectuons de façon principale, ainsi que le montrent les recherches les plus récentes en psychologie cognitive ${ }^{1}$.

La généralisation de l'ordinateur et du Web nous ayant fait entrer dans l'ère de l'écriture informatique et réticulaire, selon l'expression de

${ }^{1}$ Maryanne Wolf, Proust and the Squid. The Story and Science of the Reading Brain, New York, HarperCollins, 2007. Voir notamment le chapitre 2, « How the Brain adapted itself to read ». 
Clarisse Herrenschmidt ${ }^{2}$, il importe de se demander en quoi cette nouvelle écriture va modifier notre activité de lecteur. Tout comme dans le Phèdre Platon s'était inquiété des effets que l'écriture pourrait avoir sur la mémoire, il y a lieu de s'interroger sur les modifications que l'ordinateur va entraîner dans notre rapport à la lecture. Selon divers indices, les pratiques de lecture seraient déjà en train de se modifier sous l'effet du nouvel environnement créé par les technologies de l'information et de la communication.

Le remplacement appréhendé du livre par l'hypertexte, d'abord annoncé par le visionnaire Ted Nelson ${ }^{3}$, est devenu une réelle possibilité avec la mise en place du Web, au début des années 90. Ivan Illich, qui en plus d'être spécialisé dans l'histoire de la lecture au Moyen-âge possédait un sens aigu des réalités culturelles, reconnaissait dès alors que le livre avait cessé d'être la métaphore dominante du savoir et du sens:

Un bulldozer se cache dans tout ordinateur, qui promet d'ouvrir des voies nouvelles aux données, substitutions, transformations, ainsi qu'à leur impression instantanée. Un nouveau genre de texte forme la mentalité de mes étudiants, un imprimé sans point d'ancrage, qui ne peut prétendre être ni une métaphore ni un original de la main de l'auteur. Comme les signaux d'un vaisseau fantôme, les chaînes numériques forment sur l'écran des caractères arbitraires, fantômes, qui apparaissent, puis s'évanouissent. De moins en moins de gens viennent au livre comme au port du sens ${ }^{4}$.

C'est tout le rapport au texte qui est mis en question par l'ordinateur. Au lieu d'être incorporé à un support qui en marque clairement les frontières - comme dans le cas du livre ou du journal - le texte numérique est dématérialisé et susceptible de se couler dans n'importe quel format, souvent sans forme stable ni limites visibles, fragment d'un grand Livre où les textes de tout genre cohabitent avec le son, les images et les jeux.

L'écran ne s'annonçait pourtant pas comme un réel concurrent du papier pour l'activité de lecture, en raison notamment de sa brillance, qui est fatigante pour les yeux, de la piètre portabilité de l'ordinateur standard et de la posture qu'il impose à l'usager. Surtout, la barre de défilement du fureteur ne permet pas au lecteur de retrouver facilement l'endroit précis où il avait arrêté sa lecture précédente, ce qui est essentiel dans le cas d'un texte défilant sur une très longue " page ». En raison de ces inconvénients, l'usager d'un ordinateur va généralement se livrer à

\footnotetext{
2 Clarisse Herrenschmidt, Les trois écritures. Langue, nombre, code, Paris, Gallimard, Coll. NRF, 2007.

3 T. H. Nelson, Dream Machines : New Freedoms through Computer Screens - A minority report, Chicago, 1974.

${ }^{4}$ Ivan Illich, Du lisible au visible : Ia naissance du texte : un commentaire du Didascalicon de Hugues de Saint-Victor. Paris, Cerf, 1991, p. 141.
} 
diverses formes d'activité sur son écran, mais il est peu probable qu'il se plonge dans la lecture d'un roman. Or, le roman est le genre qui avait le plus profité de l'expansion de l'imprimerie au point qu'il en était venu à symboliser le livre et l'activité de lecture par excellence.

De fait, les changements dans les habitudes de lecture commencent à être documentés par diverses études. Aux États-unis, en 2007, un rapport de la NEA (National Endowment for the Arts) établissait que les Américains consacrent de moins en moins de temps à la lecture, que «presque la moitié des Américains âgés de 18 à 24 ans ne lisent pas de livres pour le plaisir » et que les résultats aux tests de lecture continuent à se dégrader 5 . Même si une enquête subséquente ${ }^{6}$ signalait une légère remontée de la lecture d'œuvres littéraires, il n'en reste pas moins que des enquêtes récentes effectuées en France ${ }^{7}$ et en Europe ${ }^{8}$ donnent des résultats similaires, mettant en évidence la montée d'une nouvelle "culture de l'écran », surtout chez les jeunes générations.

Ces données conduisent à se demander si la lecture ne serait pas menacée dans ses caractéristiques essentielles. Pour Christine Rosen ${ }^{9}$, le mot lire ne conviendrait même pas pour désigner l'activité que l'on fait sur un écran car ce genre de lecture serait bien inférieur à celle qui se fait avec un roman. Ce jugement de valeur repose sur une comparaison entre la lecture sur écran et la lecture romanesque. Alors que l'écran encourage une forme de perception de soi basée sur l'interaction et soumise au feedback d'autrui, récompensant « la participation et la performance, non la contemplation », le lecteur de romans se trouve dans une situation apparemment passive mais qui le rend ouvert aux expériences des autres et aux complexités de la vie.

Cette affinité entre lecture romanesque et développement d'une vie intérieure a été soulignée par divers auteurs, qui ont mis en évidence le travail d'exploration de son monde intérieur que le lecteur d'un roman est invité à faire. Le roman est en effet à même de communiquer quantité d'expériences de vie que le lecteur peut faire en imagination, l'aidant ainsi à prendre une plus grande conscience de son individualité — ce qui est

\footnotetext{
5 To Read or Not To Read: A Question of National Consequence. Research Division Report 47, National Endowment for the Arts, novembre 2007. Ce rapport avait été précédé en 2002 par Reading at Risk: A Survey of Literary Reading in America, Research Division Report 46, National Endowment for the Arts.

62008 Survey of Public Participation in the Arts. Report \# 49.

7 Voir Olivier Donnat, Les pratiques culturelles des Français à l'ère numérique, enquête 2008, éd. La Découverte/ Ministère de la culture, 284 p.

${ }^{8}$ Voir aussi « Les capacités de lecture de jeunes reculent en Europe », La Croix, $1^{\text {er }}$ décembre 2009, http://www.la-croix.com/Les-capacites-de-lecture-des-jeunes-de-15-ansreculent-en-Eu/article/2404156/25692;

9 « People of the screen », The New Atlantis, Automne 2008.

http://www.thenewatlantis.com/publications/people-of-the-screen
} 
surtout important dans les années critiques de la formation ou dans les moments difficiles de la vie. L'histoire du roman témoigne d'ailleurs d'une tendance croissante vers un récit écrit à la première personne et prenant en compte les pensées les plus intimes du narrateur, comme le montrent notamment les travaux d'Eric Auerbach ${ }^{10}$ et de David Lodge ${ }^{11}$. La neurologue Susan Greenfield ${ }^{12}$ va même jusqu'à établir un lien entre la lecture de romans et la montée de la conscience individuelle. Il est vrai que, au cours d'une lecture qui peut s'étaler sur des jours voire des semaines ou même des mois, le lecteur du roman se crée un univers imaginaire dans lequel il peut se réfugier à volonté et où s'établit une sorte de chambre de résonance entre son moi profond et celui des personnages du roman. Cela explique l'énorme pouvoir de transformation psychologique du roman pour le meilleur comme pour le pire - un pouvoir quavait bien perçu l'institution de l'Index et que Flaubert a mis en scène dans Madame Bovary. En outre, pour Jane Smiley, le roman développerait chez le lecteur à la fois des qualités d'empathie, en l'amenant à comprendre les personnages à travers le prisme de la sensibilité de l'auteur, et des qualités de sympathie, en lui faisant éprouver les peines et les joies des personnages ${ }^{13}$.

Or, ainsi qu'on l'a vu plus haut, les efforts pour adapter la lecture du roman à l'écran d'ordinateur n'ont donné jusqu'à présent que des résultats mitigés, tant cet environnement est peu compatible avec le mode de lecture au long cours que requiert le narratif. Outre les obstacles déjà énumérés, le défi principal auquel se heurte la lecture longue qu'exige le roman est la fascination que suscite l'écran d'ordinateur en tant qu'espace de flux et lieu privilégié de socialisation, où l'usager est constamment sollicité par le média: arrivée d'un courriel, d'un message Twitter ou d'une notification Facebook, séance interactive sur Skype, survol des derniers événements dans Google News, rapide coup d'œil aux derniers vidéos de YouTube, et ainsi de suite. Et tout cela sous le double impératif d'être toujours connecté et de répondre immédiatement aux sollicitations extérieures. Une telle dynamique se situe à l'opposé de celle du livre imprimé, qui invite à s'isoler du monde extérieur pour mieux s'absorber dans le fil d'un essai ou d'une fiction. Alors que le roman avait entrainé une montée de l'individualisme, voire de l'introversion, la culture de l'écran invite à une hypersocialisation - mais tout en tenant l'Autre à distance, grâce à l'absence de contact et au caractère impersonnel de l'écriture au clavier.

Le monde numérique offre ainsi un environnement propice à un état de distraction permanente que des recherches ont tenté de chiffrer statis-

\footnotetext{
${ }^{10}$ Eric Auerbach, Mimésis : la représentation de la réalité dans la littérature occidentale. Traduit de l'allemand par Cornelius Heim. Paris, Gallimard, 1968.

${ }^{11}$ David Lodge, Consciousness \& the novel : connected essays, Londres, Penguin, 2002.

${ }^{12}$ Susan Greenfield, The Quest for Meaning in the 21st Century, Londres, Sceptre, 2008.

${ }^{13}$ Jane Smiley, 13 ways of looking at the novel, New York, Knopf, 2005, p. 175.
} 
tiquement: l'usager moyen changerait d'activité toutes les trois minutes (par exemple, lecture du courriel ou de messages texte) ou passerait d'un projet à un autre toutes les 10 minutes $^{14}$. Le zapping qui caractérisait le spectateur de télévision a maintenant gagné l'écran d'ordinateur.

Si la télévision a été décrite comme une fenêtre sur le monde, s'asseoir devant un écran d'ordinateur équivaut à se retrouver tantôt dans son bureau, tantôt avec des amis à la terrasse d'un café où les gens n'arrêtent pas de passer et d'offrir le spectacle de leur diversité. Alors que le livre se caractérise par son objet étroitement défini et son unité interne, et que le roman se présente comme une totalité supposant une lecture allant de la première à la dernière page, l'écran invite le lecteur à une quête fragmentée et centrifuge, sans ordre pré-établi.

En dépit de cela, l'ordinateur est le support principal de toute lecture étroitement ciblée, où le lecteur cherche une réponse à une question : encyclopédies, dictionnaires et guides pratiques de toute sorte. Les journaux et magazines se sont aussi parfaitement adaptés à l'écran — souvent au détriment de leur contrepartie imprimée —, grâce à la mise au point d'environnements favorables : mise en page rivalisant avec celle des livres imprimés, polices de caractère adaptées à la mise en pixels, pagination et flèches de déplacement permettant un défilement horizontal plutôt que vertical. Encore plus significatif, le web est devenu en moins de 15 ans le support souvent exclusif des quelque 2500000 articles scientifiques que publient annuellement 25000 revues de par le monde.

Ayant développé des stratégies d'écrémage textuel adaptées à la vitesse des perceptions visuelles et à la fluidité du nouveau média, les usagers réclament autant de contrôle que possible sur leur lecture. Cela est surtout vrai des jeunes générations, qui ont pleinement adopté le monde numérique parce qu'il offre une quantité inépuisable de textes accessibles de partout, dans lesquels il est facile d'aller directement aux passages contenant les informations recherchées et que l'on peut partager avec d'autres en quelques clics de souris. Comme l'écrit Hubert Guillaud : « La structure web, elle, propose un autre contrat de lecture. Chaque partie de document est citable, anotable, commentable, accessible, indexable, cherchable, mixable, scriptable... De page en page, de billets en billets, de flux en flux, ces contenus sont agrégables et peuvent proposer des œuvres finalisées, consommables comme on le souhaite. »15.

Cette fluidité et cette polyvalence du média n'ont pas que des effets positifs. Étant constamment sollicitée par la récompense sociale que promet un flot incessant de messages, l'attention se disperse dans une effervescence enivrante. Beaucoup d'usagers deviennent dépendants de cette

${ }^{14}$ Gloria Mark, « Does the brain like ebooks? », The New York Times, 14 octobre 2009. http://roomfordebate.blogs.nytimes.com/2009/10/14/does-the-brain-like-e-books/

15 « Le flux est l’avenir du livre », http://lafeuille.homo-numericus.net/, 3 décembre 2009. 
drogue que leur procure le sentiment d'être connectés et il n'est pas rare que des adolescents ou même des adultes deviennent cyberdépendants au point de se réveiller la nuit pour consulter les derniers messages arrivés sur leur téléphone portable ${ }^{16}$.

L'écrit avait jusqu'ici été caractérisé par sa stabilité, sa permanence et sa parfaite adaptation à une communication différée, le plus souvent indifférente à la nature des lecteurs et à la temporalité. Sous l'effet de l'immédiateté des échanges, il est en train de perdre ces caractéristiques et de se mouler sur l'oral et la conversation. Or, cette dernière relève d'un tout autre régime cognitif que la lecture au sens classique du terme. Dans un texte célèbre, Proust compare la subtile alchimie qui prend place dans la conscience du lecteur avec la superficialité de la conversation : « la lecture, au rebours de la conversation, consist[e] pour chacun à recevoir communication d'une autre pensée, mais tout en restant seul, c'est-à-dire en continuant à jouir de la puissance intellectuelle qu'on a dans la solitude et que la conversation dissipe immédiatement, en continuant à pouvoir être inspiré, à rester en plein travail fécond de l'esprit sur lui-même »"${ }^{17}$. Ce travail fécond de l'esprit sur lui-même ne pourrait plus se faire dès lors que l'attention est émiettée par le flux incessant des événements survenant sur l'écran : messages clignotants, sons signalant une alerte ou une mise à jour logicielle, déferlement d'images, réorganisation des icônes, etc.

Dans un article intitulé "Is Google making us stupid?"18, le cybercritique Nicholas Carr se lamente de ne plus être à même de faire de la lecture « en profondeur » comme il en avait l'habitude: «ma concentration maintenant commence à dériver après deux ou trois pages. Je deviens impatient, perds le fil, commence à chercher quelque chose d'autre à faire. Je me sens comme si j'étais toujours en train de ramener au texte mon cerveau volage. La lecture profonde (deep reading), qui venait jadis naturellement, est devenue une épreuve. » Constatant que ce problème était partagé, Carr en conclut que « plus on utilise le Web, plus on doit lutter pour rester concentré sur de longs textes ». Ailleurs, il ira même jusqu'à déclarer que, puisqu'on ne lit plus de façon linéaire, « la linéarité de la raison se fond dans la non-linéarité de l'impression $»^{19}$.

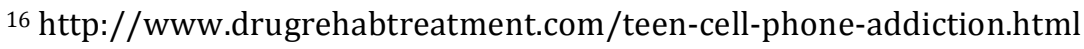

17 Proust, Marcel, 1993. Journées de lecture. Paris : UGE, collection 10/18, p. 34.

18 The Atlantic, July/August 2008. http://www.theatlantic.com/doc/200807/google

19 "Contemplative Man, the fellow who came to understand the world sentence by sentence, paragraph by paragraph, is a goner. He's being succeeded by Flickering Man, the fellow who darts from link to link, conjuring the world out of continually refreshed arrays of isolate pixels, shadows of shadows. The linearity of reason is blurring into the nonlinearity of impression; after five centuries of wakefulness, we're lapsing into a dream state." http://www.britannica.com/blogs/2008/07/this-is-your-brain-this-isyour-brain-on-the-internetthe-nick-carr-thesis/
} 
Ces appréhensions rejoignent des inquiétudes soulevées par ailleurs. Dans un ouvrage sur l'étude du cerveau en situation de lecture, Proust and the Squid, la psychologue américaine Maryanne Wolf se demande « ce qui serait perdu pour nous si on remplaçait les habiletés mises au point par le cerveau lisant avec celles en train d'être formées dans notre nouvelle génération de digital natives qui fixent leur écran avec fascination ${ }^{20}$. Ainsi qu'on l'a dit plus haut, le mode de lecture web tend en effet à reformater nos circuits cognitifs de façon à les rendre performants pour la lecture écran, avec le résultat que, placés devant une tâche de lecture relevant de l'ancien paradigme, nous avons à faire un effort de réadaptation qui peut être senti comme plus ou moins pénible.

L'idéal serait certes de maintenir bien vivants ces deux modes de lecture. Le mode par défaut est sans conteste aujourd'hui celui que commande l'écran d'ordinateur : c'est vers lui qu'on se tourne en premier lieu, pour les raisons déjà énumérées. Mais ce mode peut-il coexister avec l'ancien mode par défaut que fut la lecture du roman, qui domina au cours des trois derniers siècles, et qui est maintenant en position de repli?

S'il réussit à garder sa pertinence dans le nouveau contexte culturel, le roman pourrait certes bénéficier de la généralisation de liseuses performantes à base de papier électronique et capables de conserver des traces des lectures effectuées. Ces nouveaux supports du texte ont l'avantage d'être légers, de pouvoir stocker des bibliothèques entières, de ne pas fatiguer les yeux, de permettre au lecteur d'ajouter des annotations et de retrouver facilement la page où il s'était arrêté lors de la séance précédente. En raison de ces qualités, le marché de ces appareils est actuellement en train d'exploser. Le consommateur a maintenant le choix entre le Cybook, le Sony Reader, le Kindle, l'lliad, le Nook, le Skiff Reader, les tablettes de Plastic Logic, Microsoft, Dell, Lenovo, Apple ou encore celle de Google sur Androïd. Diverses technologies promettent pour bientôt l'affichage en couleur et la vidéo sur des supports flexibles faits de minces feuilles d'acier inoxydable. Grâce à ces tablettes adaptées à la lecture au long cours, un sentiment nouveau de confiance est en train d'apparaître chez les patrons des grandes maisons d'édition ${ }^{21}$. En fait, la plupart des obstacles que l'ordinateur opposait à la lecture suivie de longs textes ont ici disparu. $\mathrm{Si}$, en plus, ce codex électronique s'ouvrait sur une double page, comme dans les modèles annoncés par Asus et Entourage, ou mieux encore s'il était assemblé à la façon d'un codex électronique de 48 pages ou plus ${ }^{22}$, on retrouverait la possibilité de lire en profitant des possibilités

\footnotetext{
${ }^{20}$ Maryanne Wolf, Proust and the Squid. The Story and Science of the Reading Brain, New York, HarperCollins, 2007, p. 221.

${ }^{21}$ Voir notamment la présentation de François Gèze, des éditions La Découverte, sur le site http://www.enssib.fr/bibliotheque-numerique/document-48048

22 Voir la proposition que j'ai faite dès 2004 :

http://interdisciplines.org/defispublicationweb/papers/7/version/original
} 
du numérique, mais dans un environnement permettant de circuler rapidement à travers un grand nombre de pages, protégé du bruit ambiant, stable, et où les ouvrages ont fait l'objet d'un choix et restent toujours accessibles, tout comme les traces de lecture. Voilà qui assurerait une certaine viabilité à un mode de lecture encore essentiel pour qui veut s'assimiler une pensée complexe ou s'immerger durant des semaines dans une œuvre d'imagination.

Par ailleurs, il faut préciser que, en dépit des critiques que s'attire le nouveau paradigme de lecture sur écran, celui-ci présente d'énormes avantages et répond à nos besoins bien mieux que ne saurait le faire le livre imprimé, inéluctablement dépassé. La nostalgie n'est pas un guide sûr quand il s'agit d'analyser les tendances lourdes d'une civilisation. En fait, la façon dont on lit s'est constamment modifiée au fil des siècles. La lecture continue de longs romans, qui a atteint des sommets au XIXe siècle et au début du XXe, n'était certes pas le mode préféré de Montaigne, ainsi qu'on peut en juger par la citation mise en épigraphe de cet article. Comme le note Claire Bélisle en réponse aux inquiétudes exprimées par Nicholas Carr : «Le désarroi de ceux qui voient se creuser l'écart entre la lecture et la concentration rappelle étrangement les réprobations de lettrés du XVIIe siècle, qui ne supportaient pas que l'on puisse envisager de lire sans méditer $»^{23}$.

Il faut aussi reconnaître que l'attention est particulièrement en éveil quand le lecteur ne se contente pas de suivre le fil du texte mais en évalue la pertinence par rapport à ses objectifs. Habitué à faire des choix dans la navigation, le lecteur apprend en effet à développer un jeu d'habiletés cognitives caractéristique de ce que je propose d'appeler une lecture " ergative », c'est-à-dire une lecture orientée vers le travail et l'action. Ce genre de lecture existe depuis toujours, mais elle fut longtemps le propre d'une petite élite de savants ou d'écrivains qui lisaient d'abord en vue d'extraire de leurs lectures des données précises au service d'une recherche personnelle. C'est pour répondre aux besoins de cette classe intellectuelle que les index s'étaient répandus dans les éditions parisiennes à partir du XIIIe siècle 24 .

Avec l'ordinateur, ce mode de lecture est accessible à tout un chacun, car l'indexation intégrale et le remplacement du fil continu du discours par une structure en base de données favorise une lecture entièrement commandée par les intérêts de l'usager. Le fait que tous les éléments d'information contenus dans des milliards de pages soient accessibles en

\footnotetext{
${ }^{23}$ Claire Bélisle, «La nouvelle frontière de la connaissance numérique », Lecture, rêve, hypertexte, sous la dir. de Rainier Grutman et Christian Milat, Ottawa, Éd. David, 2009, p. 194.

${ }^{24}$ Voir Mary \& Richard House, "La naissance des index ", Histoire de l'édition française, tome I, sous la direction de Henri-Jean Martin et Roger Chartier, Paris, Promodis, 1982 , p. $77-86$.
} 
quelques fractions de seconde ouvre des horizons nouveaux aux questions que l'on peut poser à des textes et permet de les aborder de la même façon que Claude Lévi-Strauss interrogeait les mythes: lecture croisée de tous les textes référant à un même lieu à travers l'histoire, à un même nom propre, repérage d'un intertexte commun ou des divers avatars qu'ont connus les représentations culturelles au fil des siècles. C'est en fonction de ces nouveaux modes de lecture, qui procèdent par repérage de séries et de paradigmes communs, qu'il faut évaluer l'entreprise de Google Books, où sont déjà numérisés plus de dix millions de livres. De toute évidence, la numérisation donnera à ces ouvrages une vie nouvelle car ils pourront être mis en résonance entre eux : une recherche sur un mot ou une expression fera apparaître des filiations textuelles insoupçonnées, un même nom propre rapprochera des ouvrages écrits dans une variété de langues. Déjà, Google offre des outils d'analyse en mode Beta permettant d'afficher les résultats de recherche en catégorisant de façon visuelle les domaines principaux auxquels se rattachent les pages de la recherche (Wonder Wheel) ou en les reportant sur un graphe temporel (Timeline). La mise en commun des annotations pourrait à terme relier l'ensemble de la bibliothèque virtuelle par des liens hypertexte, selon la suggestion de Kevin Kelly 25 .

Grâce à ces outils de recherche, nous nous trouvons, pour la première fois dans l'histoire, dans la position où des réponses sont aisément disponibles pour la plupart des questions de type scientifique ou documentaire que l'on se pose couramment. Or, s'il est avéré que la curiosité intellectuelle se développe dans la mesure où l'individu est plongé dans un environnement intellectuellement stimulant, il est à prévoir que le nouvel écosystème cognitif entraînera un essor prodigieux de la curiosité intellectuelle et de la créativité. Ce qui avait fait le pouvoir d'attraction de villes comme Athènes, Alexandrie ou Paris, est maintenant disponible à l'humanité entière. Avec l'Internet, les idées sont de plus en plus assimilables à ces mèmes que Richard Dawkins définit comme des êtres vivants, avides de se reproduire et susceptibles d'évoluer par variations et sélection naturelle ${ }^{26}$.

Ce n'est pas seulement notre façon de lire qui sera transformée par l'ordinateur, mais tous les aspects de la cognition. Depuis quelques dizaines d'années, déjà, nous avons perdu l'habitude de mémoriser des textes ou des données. Ce phénomène s'est accentué avec l'arrivée du Web, qui a entraîné une externalisation massive de notre mémoire, maintenant prise

\footnotetext{
${ }^{25}$ Kevin Kelly, «Scan this book! », The New York Times, 14 mai 2006. Cette suggestion avait suscité une réponse indignée de John Updike le 25 juin dans le même journal sous le titre «The end of authorship ».

${ }^{26}$ Richard Dawkins, The Selfish Gene, Oxford: Oxford University Press. 1976.
} 
en charge par les moteurs de recherche ${ }^{27}$ ou par des outils d'enregistrement de nos moindres faits et gestes à la façon de Gordon Bell, cet ingénieur de Microsoft qui archive la totalité de son vécu ${ }^{28}$. C'est vers Google ou l'index de notre ordinateur que nous nous tournons désormais pour retrouver le nom d'un endroit où nous sommes passés, celui d'une personne que nous avons rencontrée, ou d'un texte que nous avons lu. Un fragment infime est souvent suffisant pour ramener à la surface l'élément oublié. Grâce à cette mémoire virtuelle globale toujours à notre disposition, nous évitons maintenant de nous encombrer de myriades de détails qui, jadis, auraient caractérisé un esprit cultivé et qui faisaient les délices de la conversation. Comme une bonne part de nos échanges se fait en ligne, cette délégation de notre mémoire à une instance électronique ne pose guère de problème: en quelques clics de souris, l'information recherchée est retrouvée, copiée/collée et acheminée à notre correspondant. Dans le cas des rencontres physiques, il n'est pas rare de voir les interlocuteurs effectuer, tout en parlant, des recherches frénétiques dans leur téléphone portable ou leur iPod afin de suppléer à une mémoire défaillante. Celle-ci se concentre désormais sur les mots de passe à retenir et sur les procédures à suivre pour faire fonctionner les dizaines de gadgets ou d'applications informatiques que nous avons accumulés en vue de nous rendre la vie plus facile.

Mais cette externalisation de la mémoire a un coût : moins un individu a de mémoire, plus il est malléable et tend à se fondre dans le discours social ambiant. On ne saurait ignorer non plus la troublante convergence entre l'étiage de la mémoire personnelle et le remplacement du livreconteneur par le texte-flux. Dès lors que la lecture se fait au rythme de la conversation, il y a risque que le flux des échanges ne permette pas au lecteur de s'engager dans ce supplément de réflexion personnelle que Proust considérait comme l'essence de la lecture et que l'anglais appelle deep reading. Or, si la lecture ne laisse pas de trace mémorable dans l'esprit du lecteur et n'entraîne pas de réorganisation de sa compréhension de soi ou du monde, elle n'est plus qu'un jeu gratuit de collecte de signes, la communication entre les êtres se réduisant pour l'essentiel à un échange de données phatiques sous la forme d'acronymes (du genre « LOL ») ou d'émoticônes.

Avec l'externalisation de la mémoire, c'est aussi le for intérieur qui tend à se réduire. L'internaute qui obtempère quotidiennement aux sommations de Facebook («À quoi pensez-vous? ») ou de Twitter («Que faites-vous? ») en arrive à élargir la sphère de son intimité à des dizaines voire des centaines de personnes. À toute fin pratique, la vie privée est en

\footnotetext{
${ }^{27}$ Voir mon article « L'hypertexte et l'avenir de la mémoire », Le Débat, no 115, mai-août 2001 , p. $145-155$.

${ }^{28}$ Gordon Bell et Jim Gemmell, Total Recall: How the E-Memory Revolution Will Change Everything, New York, Penguin, 2009.
} 
train de devenir une illusion, laissant le champ libre aux maffias de l'informatique mais aussi à l'emprise grandissante du pouvoir sur nos vies. Comme le note Richard Foreman, en lieu et place de notre dense héritage culturel, nous sommes en train de nous transformer en "pancake people - spread wide and thin $»^{29}$.

La transformation des mœurs sous l'emprise de l'Internet illustre une fois de plus la justesse de la définition qu'Aristote donnait de l'homme comme un « animal social ». La planète est devenue une vaste ruche où les échanges entre les êtres se sont multipliés prodigieusement. Cela fait aussi de nos sociétés des proies idéales pour les groupes identitaires dont le fanatisme se renforce à se voir partagé dans des communautés virtuelles.

$* * *$

Au moment de conclure, je reconnais volontiers que ma pensée dans cet article est marquée par une oscillation entre le nouveau et l'ancien paradigme. En un peu plus de deux millénaires, notre civilisation a accompli en matière d'écriture un cercle complet - plus précisément : une révolution. Avec Platon, elle avait commencé par dénigrer l'écriture pour exalter les vertus de la conversation, seule voie selon Socrate pour accéder à la vérité. Mais l'écriture avait triomphé et nul écrivain n'illustre mieux ce triomphe que Marcel Proust, l'homme du livre par excellence, qui aurait aimé voir publier La Recherche du temps perdu en un seul volume, et pour qui la conversation ne pouvait que maintenir à la surface des choses. Avec le numérique, nous sommes en face d'un média inédit, qui transforme radicalement notre rapport traditionnel à l'écrit : de la fixité du papier, le texte passe dans le régime du flux; au lieu d'une lecture déférente et respectueuse du fil linéaire donné par l'auteur à son discours, le texte est appréhendé par des moteurs de recherche qui ciblent très précisément les éléments recherchés et les groupent en séries; au lieu d'un auteur unique, le texte est issu de voix multiples qui s'entrecroisent, s'additionnent ou se corrigent mutuellement; à la célébration de l'auteur inspiré par les muses, fait place l'anonymat — le renoncement à la signature permettant par ailleurs le développement prodigieux de Wikipédia et des programmes open source. Plus globalement, la domination millénaire du logos est en train de reculer sous la montée du visuel, qui invite à une perception immédiate et synthétique - mais aussi plus apte à communiquer une charge émotive diffuse, aux dépens des capacités d'analyse.

Le défi est de faire en sorte que, par une éducation précoce et un entrainement adéquat, la jeune génération s'assimile les outils intellectuels permettant à chacun de moduler sa lecture selon ses besoins : lecture savante avec prise de notes, lecture documentaire avec classement et hiérarchisation des sources, lecture plaisir, et lecture-écriture participative dans le flux du web. Ce

${ }^{29}$ Cité par Nicholas Carr, The Big Switch, p. 227. 
faisant, on arriverait à dépasser la fascination que suscitent les nouveaux outils numériques et à les utiliser non pas pour le plaisir de les voir fonctionner, mais pour leur valeur instrumentale et leur apport à notre besoin d'informations et à la construction du moi intime et du moi social. 